\title{
Sobre Bathysciinae catalanes (Col. Catopidae) $)^{1}$
}

\author{
Por F. Español $\left.{ }^{2}\right)$ \\ Col laminas 1(1)-2 (2)
}

Como consecuencia de la intensa actividad desplegada por diferentes equipos barceloneses de exploraciones subterráneas, estos últimos años han sido pródigos en descubrimientos biospeleológicos en toda la región catalana. En notas anteriores, actualmente en curso de impresión, incié el estudio del material recogido con la descripción de algunas novedades y pasando revista a diversos grupos con ellas relacionados.

Continuando la mencionada labor en el presente trabajo, dedicado a los géneros Speonomus y Speophilus, me ocupo de aquellas especies, no comentadas todavía, cuya captura encierra algún nuevo dato a tener en cuenta para el mejor conocimiento de estos insectos.

Por ser tema de estudio del Sr. Escolá, el gén. Troglocharinus queda al margen del presente comentario, si se exceptúa una nota rectificativa referente a impelletierii Españ. descrito como raza dehustachei Jeann. y que hoy, con más elementos de juicio, creo debe separarse especificamente de éste.

Antes de entrar en la parte descriptiva deseo agradecer a los miembros del G.I.E., E.R.E., C.G.B., S.I.E. y E.D.E.C.A. la eficaz colaboración prestada.

Gén. Speonomus Jeann.

S. (s.str.) DELAROUZEEI subsp. CATALONICUS Jeann.

Barcelona: Avenc de Sant Ou, Montgrony, 23-IX-62 (Escolá, Senent): avenc de Can Calderer, Figols, 1-XI-63 (Escolá).

La captura de un representante del complejo delarouzeei en los alrededores de Figols tiene el interés de extender más allá del Llobregat el área de estos Speonomus y de señalar, muy posiblemente, el límite de su dispersión hacia el $\mathrm{W}$, por entrar en la referida región en contacto con el grupo del mengeli propio de esta zona, es decir de los relieves

1) Este trabajo se ha beneficiado de la ayuda concedida a la Cátedra de Zoologia (invertebrados) con cargo al crédito destinado al fomento de la investigación en la Universidad.

2) Museo de Zoologia, Apartado 593, Barcelona (3). 
comprendidos entre el Llobregat y el Segre. A partir de este último y hacia occidente aparece el grupo del crypticola que junto con sus próximos parientes los Speonomus del subgén. Speonomites colonizan las diferentes sierras comprendidas entre el Segre y ambos Nogueras.

\section{S. (s.str.) $A U R O U X I$ n.sp. (Fig. 1)}

Lérida: Escletxa Minguera, Sant Miquel de la Vall, 8-XII-64 (Auroux, Amat); id. 26-XII-64 (I. Gonzalez, Auroux, Amat, Español); escletxa de la Vinya d'en Jaufré, Sant Miquel de la Vall, 27-XII-64 (Auroux, Amat); cova de Sant Gervás, Sant Miquel de la Vall 27-XII64 (I. Gonzalez, Auroux, Amat, Español).

Long. 2,6-3,5 mm. Talla relativamente grande, cuerpo oval, convexo y atenuado por detrás. Color moreno testáceo o amarillo pálido. Pubescencia corta, fina y acostada, de un tono amarillento. Antenas largas y gráciles alcanzando ampliamente los dos tercios de la longitud del cuerpo, engrosadas a partir del $7^{\circ}$ artejo; el $8^{\circ}$ casi doble tan largo como ancho y algo más corto que el 9o; a continuación se dan las longitudes y anchuras relativas de cada uno de ellos en el $\delta^{\star}$ :

$\begin{array}{lllllllllllr}\text { Artejos } & 1^{\circ} & 2^{\circ} & 3^{\circ} & 4^{\circ} & 5^{\circ} & 6^{\circ} & 77^{\circ} & 8^{\circ} & 9^{\circ} & 10^{\circ} & 11^{\circ} \\ \text { Longitud } & 6 & 6 & 6 & 6 & 7 & 6,2 & 7 & 3,5 & 5 & 4 & 6,5 \\ \text { Anchura en } & 2 & 1,6 & 1,6 & 1,6 & 1,6 & 1,7 & 2 & 1,7 & 2,10 & 2,1 & \\ \text { el ápice } & & & & & & & & & & & \end{array}$

Cada unidad equivale a $1 / 28 \mathrm{~mm}$.

Protórax fuertemente transverso, de lados muy estrechados hacia adelante y con la máxima anchura en la base; la puntuación finísima y poco apreciable. Elitros cuneiformes, vez y media tan largos como anchos; con las estriolas transversas muy aparentes, densa y regularmente dispuestas en toda la superficie, incluida la declividad apical; estría sutural sólo indicada y a menudo difícil de apreciar.

Quilla mesosternal alta, en ángulo recto y vivo, el borde anterior convexo.

Patas robustas; tibias intermedias más espinosas que las otras; las posteriores rectas; tarsos anteriores del đô un poquitín más anchos que el ápice de las tibias (fig. 2).

Organo copulador $\widehat{o}$ con los estilos armados de 3 sedas de las que dos son grandes y fuertemente sinuosas; la tercera menor y situada en el penicilo; éste poco desarrollado (fig. 3).

Pertenece al grupo del crypticola con cuya especie presenta las máximas analogías y al lado de la cual debe colocarse. Difiere de ella por la talla media algo mayor, el cuerpo convexo (deprimido en crypticola); proporcionalmente más estrecho, por lo que parece más 
alargado; las antenas más largas y los tarsos anteriores del $\hat{o}$ más fuertemente dilatados.

Más alejado de puncticollis por la talla mayor, los élitros más fuertemente acuminados por detrás, menos redondeados en los lados; el $8^{\circ}$ artejo de las antenas poco más corto que el $9^{\circ}$; etc.

Por la armadura de los estilos podría pensarse en el subgén. Speonomites que habita la misma región, pero tanto por la escultura elitral como por el tipo de pubescencia queda al margen del referido subgénero.

Dedicado a su descubridor D. Luis Auroux del G.I.E. y asiduo colaborador de la sección biospeleológica del Museo de Zoología de Barcelona, que en poco tiempo se ha revelado como uno de los más activos y eficaces exploradores de nuestro dominio subterráneo.

\section{S. (s.str.) PUNCTICOLLIS Jeann.}

Lérida: Cova de Font de Pou, Ager, 21-V-64 algunos ejemplares (Español).

Los precedentes ejemplares parecen idénticos al puncticollis s.str. de la cova de l'Or, en el Paso dels Terradets, a cuya forma creo deben referirse.

Hasta la fecha el puncticollis s. str. era sólo conocido de dicha cova de l'Or y de la cova del Gel, situadas ambas en la Sa de Montsech de Rubies (orilla izquierda del Noguera Pallaresa). Su captura en la zona de Ager tiene pues el interés de ampliar el área de repartición del mismo a la orilla derecha del mencionado rio.

\section{S. (s.str.) ZARIQUIEYI Jeann.}

Lérida: Avenc Grallé de Badiá, Ager, 31-VIII-61 (Escolá).

La expresada cavidad se abre en el Montsech d'Ager, entre Ager y Corsá, no lejos de la cova Negra y de la cova Colomera en las que el Dr. Zariquiey recogió la serie típica de esta especie.

$$
\text { S. (s.str.) MENGELI Jeann. }
$$

Lérida: Cova de Vinyoles o dels Encantats, entre Cava y Toloriu, estribaciones septentrionales del Cadí, Cerdaña, 31-VIII-61 (Suñer).

En la misma cueva fué descubierto por los Profs. Jeannel y Racovitza, y encontrado después por el Dr. Zariquiey.

\section{S. (s.str.) MERCEDESI Zar.}

Lérida: Avenc de Coll d'Alsina, Odén, 22-IX-63 (Escolá, Subils, Senent); avenc dels Encantats, Odén, 23-IX-63 (Senent, Subils, Escolá); avene del Xato, Llinás, Oliana, 3-V-64 (Subils, Escolá, 
Senent); cova de les Encantades, Llinás, Oliana, 3-V-64 (Escolá, Senent, Subils); todas estas cavidades se abren en los relieves situados entre el Segre y Canalda, al Sur de la Sa de Odén.

En todos los ô ô examinados de las mencionadas cavidades los estilos del órgano copulador están armados de tres sedas apicales bien desarrolladas, caracter que según Jeannel, separa mercedesi de mengeli (con sólo dos, por falta de la dorsal). Ello no obstante se trata de dos formas muy próximas y con casi idéntica morfología externa.

Con anterioridad a las citadas recolecciones mercedesi era sólo conocido de la Bofia de Sant Jaume en las estribaciones meridionales de la Sa del Cadí. Las nuevas localizaciones que se acompañan extienden, pues, el área del insecto por la zona comprendida entre el Segre y el Llobregat.

\section{S. (SPEONOMITES) NITENS Jeann.}

Lérida: Cova del Tabaco, en la Sa de Montroig, sobre Camarasa (I. Gonzalez, Auroux, Amat, Español); avenc de la Presa, Sa de Montroig, no lejos de la anterior (Auroux, I. Gonzalez, Amat, Español).

Descrito y sólo conocido hasta el presente de la primera de las dos mencionadas cavidades.

Como dato complementario señalaré que en la cova del Tabaco a parte el citado Bathysciinae, raro en la gran sala central y relativamente abundante, como indica Jeannel, en una galería lateral, muy húmeda, próxima a la entrada, se recogieron los siguientes coleópteros: Akis sansi Sol. y Blaps lusitanica Hbst. debajo un montón de piedras en el vestíbulo de la cavidad; Tipnus unicolor Pill. errante por el suelo en diferentes rincones oscuros y secos de la sala central; Pristonychus terricola Hbst. debajo las piedras y en el guano depositado en el interior de la cueva; Gnathoncus nannetensis Mars. en compañia del anterior; estos dos últimos insectos habian ya sido observados con anterioridad en la cova dels Muricets situada en el paso dels Terradets (Sellés) frecuentada por quirópteros gregarios y en la que se acumula gran cantidad de murcielaguina.

\section{S. (SPEONOMITES) VELOX Jeann.}

Lérida: Queran dels Picalts, Llucás, cerca Vilanova de Meiá (Senent).

Algo menor que el precedente del que difiere, además, por el notable desarrollo de la pubescencia, larga y erizada en toda la superficie del cuerpo. 
Esta nueva cita viene en apoyo de la localización del insecto en las distintas cavidades que se abren en los alrededores de Vilanova de Meiá.

\section{Gén. Speophilus Jeann.}

$$
\text { S. FONTI s.str. }
$$

Lérida: Bofia de la Matella de les Planes, Vall de Cabó, en el extremo oriental de la $\mathrm{S}^{\mathrm{a}}$ del Boumort (Escolá, Senent).

La presente captura amplía un poco hacía el norte el área de este Speophilus, sólo señalado hasta el presente de la cova d'Armini, Montanisell, entre Organyá y Coll de de Nargó.

\section{S. FONTI subsp. SCHUTTEI Españ.}

Lérida: Forat de la Roqueta, Ortoneda, en el extremo occidental de la $\mathrm{S}$ a del Boumort (Victoria).

De esta raza se conocía sólo la serie típica, recogida en la cova Font Mentidora de Peralba, situada en la misma región de la $\mathrm{S}^{\text {a }}$ del Boumort.

$$
\text { S. SUBILSI n.sp. (fig. 4) }
$$

Lérida: Grallera de Cambrils, Llinás, Oliana, en la $\mathrm{S}^{a}$ de Odén (Escolá, Senent); avenc de l'Obaga del Grau, Alsina d'Alinyá, estribaciones septentrionales de la $\mathrm{S}^{a}$ de Odén (Subils).

Long. 2,5-2,8 mm. Cuerpo largamente elíptico, apenas más atenuado por detrás que por delante y deprimido por encima; pubescencia fina, densa y acostada; escultura regular formada de puntos muy finos y apretados sobre el pronoto y de estriolas transversas, densamente dispuestas sobre los élitros.

Antenas gráciles, moderadamente alargadas, poco ensanchadas en su parte terminal, alcanzando los $3 / 4$ de la longitud del cuerpo, $8^{\circ}$ artejo tan largo como el $9^{\circ}$; las longitudes y anchuras proporcionales de los artejos en el ơ son:

$\begin{array}{llllllllllll}\text { Artejos } & 1^{\circ} & 2^{\circ} & 3^{\circ} & 4^{\circ} & 5^{\circ} & 6^{\circ} & 7^{\circ} & 8^{\circ} & 9^{\circ} & 10^{\circ} & 11^{\circ} \\ \text { Longitud } & 5,5 & 5 & 5 & 6 & 7 & 6 & 6 & 4,8 & 4,8 & 4,6 & 7 \\ \begin{array}{l}\text { Anchura en } \\ \text { el ápice }\end{array} & 2 & 1,4 & 1 & 1 & 1 & 1 & 2,2 & 1 & 2,2 & 2,2 & \end{array}$

Cada unidad equivale a $1 / 28 \mathrm{~mm}$.

Protórax poco transverso, con la máxima anchura en la base; a partir de ésta los lados se estrechan apenas y se hacen ligeramente sinuosos hasta la mitad de su longitud, para estrecharse luego fuerte- 
mente y casi en línea recta hasta el borde anterior; base ligeramente bisinuosa; ángulos posteriores un poco agudos, subrectos.

Élitros alargados, casi doble tan largos como anchos, fuertemente atenuados en la mitad apical, algo deprimidos a lo largo de la sutura y con la estría sutural apenas indicada; redondeados por separado en la extremidad.

Quilla mesosternal alta, formando un ángulo casi recto y con el vértice ganchudo.

Patas gráciles; tibias intermedias sinuosas; las posteriores rectas; tarsos casi tan largos como sus respectivas tibias; los anteriores del $\vec{\sigma}$ apenas más estrechos que la parte terminal de las tibias.

Órgano copulador masculino conforme al tipo del género; estilos armados en la zona apical de tres grandes sedas, dos ventrales y una dorsal, asociadas a un mechón de pelos largos y densamente dispuestos (fig. 5).

Dimorfismo sexual bastante manifiesto; en la + el cuerpo es algo más grande y robusto, las antenas apenas más cortas y los tarsos anteriores simples.

Morfológicamente se sitúa en la proximidad de carrodillae Jeann. de la Grallera de Estadilla (Huesca) que sólo conozco por la descripción. Pero a parte la regular distancia que le separa de éste en la que se intercalan los cursos del Segre y de ambos Nogueras, y de estar los relieves comprendidos entre la sierra de Odén y la de Carrodilla habitados por otros Speophilus, existen sensibles diferencias entre ambos insectos que apoyan su independencia específica. En efecto, subilsi es de talla sensiblemente menor, su contorno es más largamente elíptico, la pubescencia está en él bien desarrollada y densamente dispuesta; las antenas son más cortas y sus élitros están deprimidos a lo largo de la sutura.

Todavía más diferente de fonti Jeann. y de quadricollis Jeann. por la talla menor, el cuerpo más atenuado lo mismo por delante que por detrás; el protórax con la máxima anchura en la base; los lados ante ésta menos paralelos y apenas sinuosos, más fuertemente estrechados en su mitad anterior; las antenas más cortas; en fin, por los élitros menos abombados lateralmente, más acuminados hacia atrás y redondeados por separado en la extremidad.

No precisa compararlo con cenarroi Españ. ni con las formas del grupo kiesenwetteri por estar muy alejado morfológicamente de todos ellos. 
Gén. Troglocharinus Rtt.

\section{T. IMPELLETIERII Españ.}

Lérida: Cova La Palomera, Tahús, en la confluencia del torrente de Tahús con el de Castell (Español).

Aunque descrito como raza de hustachei se trata, en realidad, de una buena especie, como he podido comprobar a la vista del ô del citado hustachei que me seguía desconocido, capturado recientemente por los Sres. Auroux y Amat en una sima situada junto a la cova del Gel, Montsech de Rubies, (Lérida) de donde procede la serie típica de este insecto.

El estudio comparado de ambos Troglocharinus permite, en efecto, observar sensibles diferencias en el contorno del protórax y élitros, en la longitud de las antenas, en el desarrollo de la quilla mesosternal y en la dilatación de los tarsos anteriores del $\widehat{\jmath}$, que justifican la independencia específica de impelletierii.

El detalle de las expresadas diferencias aparecerá en el trabajo que prepara el Sr. Escolá sobre el gén. Troglocharinus.

\section{RESUMEN}

Enumeración de los Speonomus y Speophilus (Col. Catopidae) recogidos por diferentes espeleólogos relacionados con el Museo de Zoología de Barcelona, en una serie de cavidades la mayor parte de las cuales seguian inexploradas y sin dato alguno sobre su población de Catopidae. Con ello no se persigue otro objetivo que el de ampliar nuestros conocimientos sobre la fauna cavernicola de la vertiente catalana de los Pirineos.

Se acompaña la descripción de dos nuevas especies (Speonomus aurouxi y Speophilus subilsi) y un breve comentario sobre Troglocharinus impelletierii Españ. descrito como raza de hustachei Jeann., pero separado de éste por importantes diferencias morfológicas que justifican su aislamiento específico.

\section{RÉSUMÉ}

Enumération des Speonomus et Speophilus (col. Catopidae) récoltés par divers spéléologistes en relation avec le Musée de Zoologie de Barcelone, dans des cavités d'où en général aucun Coléoptère troglobie n'avait encore été cité, de sorte qu'elle complète nos connaissances sur la faune cavernicole du versant catalan des Pyrénées.

On accompagne la description de deux espèces nouvelles (Speonomus aurouxi et Speophilus subilsi), d'un bref commentaire sur le Troglocharinus impelletierii Españ. décrit comme race d'hustachei Jeann., mais différant de celui-ci par des caractères bien tranchés qui justifient son isolement spécifique. 


\section{BIBLIOGRAFIA}

Jeannel, R. (1911) - Revision des Bathysciinae. Biospeologica XIX. Arch. Zool. exp. (5) VII, p. 325-365.

- (1924) - Monographie des Bathysciinae. Biospeologica L. Arch. Zool. exp. (1) LXIII, p. 130-173.

Español, F. (1953) - Sobre Bathysciinae ibéricos: Adiciones a la última revisión del Prof. Jeannel (1924). Speleon, IV, no 1, p. 37-48.

- (1955) - Nuevos datos sobre la entomofauna cavernícola de la provincia de Lérida. Eos, XXXI, p. 261-273.

- (1956) - Los Bathysciinae catalanes. Act. 2 Congr. Intern. d'Etud. Pyrénéen. Luchon-Pau, t. 3, sect. II, p. 109-123.

\section{EXPLICACION FIGURAS LAMINA 1 (1)}

Fig. 1: Speonomus (s.str.) aurouxi n.sp. ơ.

Fig. 2: Speonomus (s.str.) aurouxi n.sp., pata anterior del ${ }_{0}$.

Fig. 3: Speonomus (s.str.) aurouxi n.sp., parte terminal del estilo del órgano copulador ${ }^{*}$.

Fig. 4: Speophilus subilsi n.sp. ơ.

Fig. 5: Speophilus subilsi n.sp., parte terminal del estilo del órgano copulador $\sigma^{*}$ : a, cara ventral; b, cara dorsal.

\section{LAMINA $2(2)$}

Esquema de la distribución de los géneros Speonomus y Speophilus en el Prepirineo catalán, zona comprendida entre los cursos del Llobregat y del Noguera Ribagorzana: 1, Speonomus (s.str.) delarouzeei ssp. catalonicus Jeann.; 2, S. (s.str.) crypticola Jeann., S. (s.str.) ellipticus Jeann.; 3, S. (s.str.) crypticola Jeann.; 4, $S$. (s.str.) aurouxi n.sp.; 5, 6 y 7, $S$. (s.str.) puncticollis Jeann.; 8 y 11, S. (s.str.) zariquieyi Jeann.; 9, S. (s.str.) puncticollis sssp. latrunculus Jeann., $S$. (Speonomites) nitens Jeann.; 10, $S$. (s. str.) puncticollis ssp. troglodytes Jeann.; 12, S. (s.str.) sancti-gervasi Jeann., $S$. (s.str.) latebricola ssp. elongatus Jeann.; 12 y 15, Speophilus quadricollis Jeann.; 13, Speonomus (s.str.) ribagorzanus Jeann.; 14, S. (s.str.) pallaresanus Jeann.; 15, S. (s.str.) latebricola Jeann.; 16, S. (s.str.) mengeli Jeann.; 17 y 18, S. (s.str.) mercedesi Zar.; 19, S. (Speonomites) colominasi Zar.; 20, $S$. (Speonomites) velox Jeann.; 21 y 22, Speophilus fonti Jeann.; 23, S. fonti ssp. zariquieyi Jeann.; 24, S. fonti ssp. infernus Jeann.; 25 y 26 , S. fonti ssp. schüttei Españ.; 27 y 28, S. subilsi n.sp.; 29, S. cenarroi Españ. 

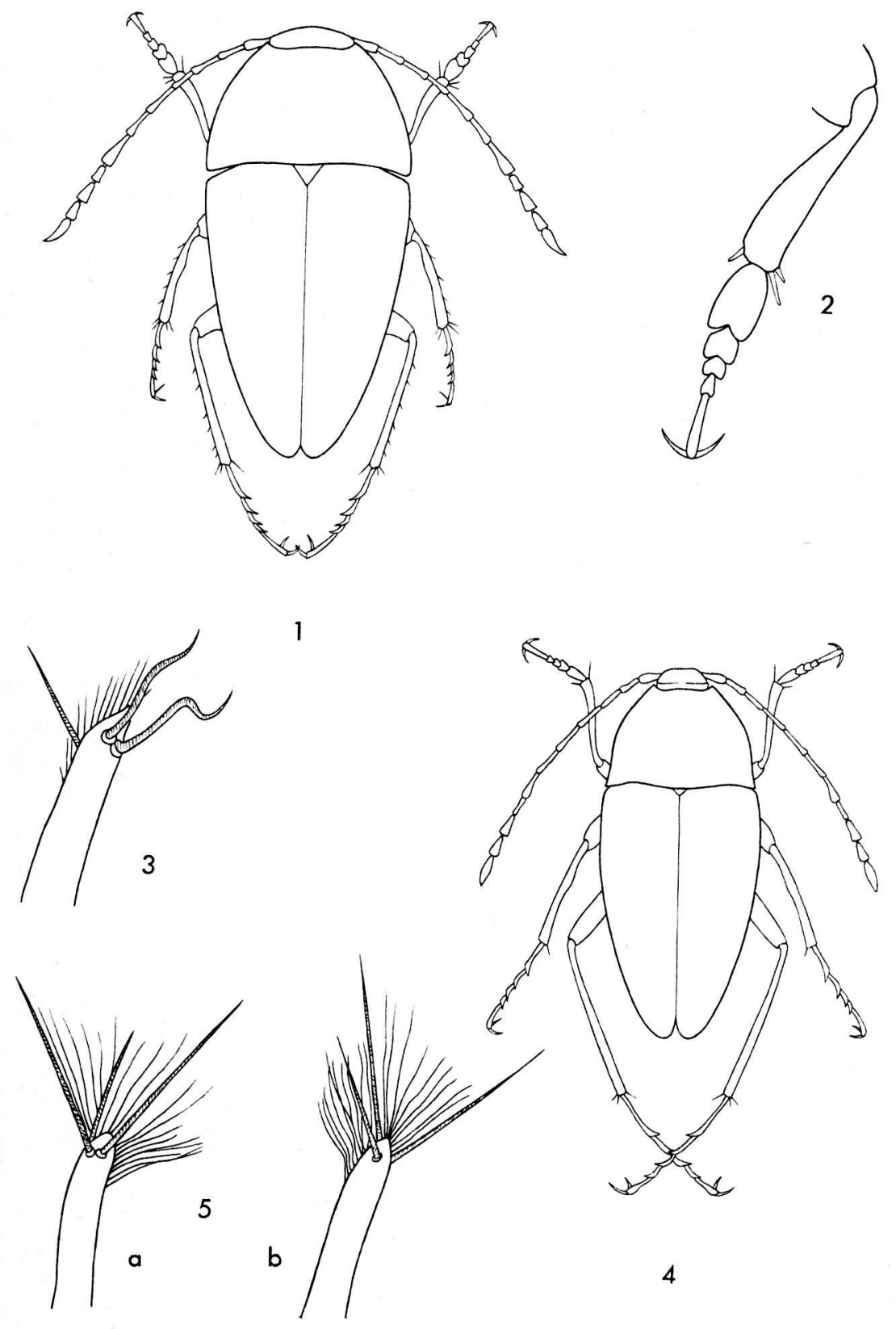


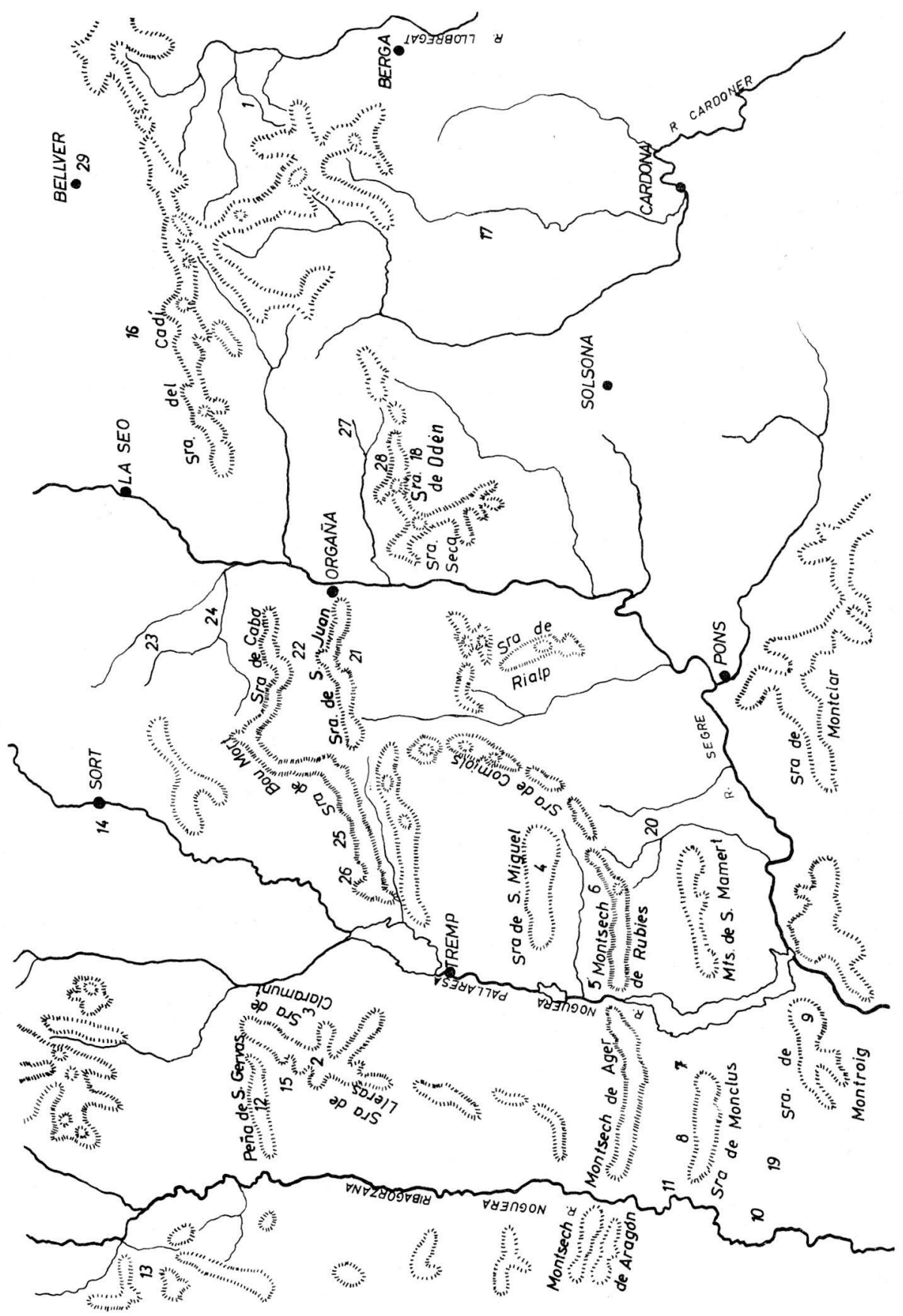

